\title{
DIFERENCIACIÓN REGIONAL Y TRANSFORMACIÓN SECTORIAL DE LA MANO DE OBRA FEMENINA EN MEXICO, 1970
}

\author{
Marta Tienda* \\ Universidad de Wisconsin-Madison
}

\section{INTRODUCCIÓN}

EXISTEN PRUEBas importantes que confirman el hecho de que la participación de la fuerza de trabajo femenina varía con los índices de desarrollo económico. ${ }^{1}$ Aunque también es cierto que las estructuras de empleo y producción sufren cambios considerables a medida que un país (o una región) se moderniza y se desarrolla económicamente. $\mathrm{Y}$ dado que los cambios en la asignación industrial de la mano de obra por sexos son parte integral de la transformación sectorial del empleo que acompaña al desarrollo, es importante considerar en qué forma los cambios en la contratación de mujeres están influidos por los cambios de empleo en los sectores de la industria." Por desgracia, hay pocos estudios de ese tipo sobre el empleo de mujeres en los países en vías de desarrollo, dado que el grueso de la investigación sobre este tema proviene de países industrializados.

Una de las características más sobresalientes de las pautas de asignación de la fuerza de trabajo femenina transculturalmente es el grado de

* Agradezco el apoyo financiero del Population Research Center (Centro de Investigación sobre la Población) de la Universidad de Texas en Austin. En particular expreso mi agradecimiento a Harley L. Browning por sus atinados comentarios y críticas a las versiones preliminares de este trabajo.

1 Naciones Unidas, Sex and Age Patterns of Participation in Economic Activities, Nueva York, Department of Economic and Social Affairs, 1962; Gertrude Bancroft, "Patterns of Female Labor Force Activity", Industrial Relations, Núm, 9, mayo de 1968, pp. 204-218; Murray Gendell y. Guillermo Rossel, "The Trends and Patterns of the Economic Activity of Women in Latin America During the 1950's", Estadistica, Núm. 26, septiembre de 1968, pp. 561-576; Joseph R. Ramos, Labor and Development in Latin America, Nueva York, Columbia University Press, 1970; National Manpower Council, Womanpower, Nueva York, Columbia University Press, 1957.

${ }_{2}$ La mayoría de los estudios que examinan los cambios en el empleo de mujeres se centra en la dimensión ocupacional más que industrial de la estructura del empleo. Véase por ejemplo, Rosemary Santana Cooney, "Female Professional Work Opportunities: A Cross-National Study", Demography, Núm. 12, febrero de 1975, pp. 107-120; Rudolph C. Blitz, "An International Comparison of Women's Participation in the Professions", The Journal of Developing Areas, Núm. 9, julio de 1975, pp. 499-510. 
concentración en ciertos empleos. ${ }^{3}$ Aun cuando existen diferencias transculturales, en general, a medida que más y más mujeres se han vuelto económicamente activas, es usual que se vean absorbidas por empleos relacionados con sus funciones domésticas tradicionales o por actividades consideradas propias para el sexo femenino. El significado de tales pautas de distribución es un mercado de trabajo cuya división se basa en el sexo del trabajador como en otras características socioeconómicas y demográficas.

Dicho en términos más extremos, en la medida en que los mercados de trabajo masculino y femenino están divididos en sectores de lucha, los hombres y las mujeres no compiten por los mismos trabajos debido a que las características de los sexos hacen también que la demanda sea específicamente sexual. Esta consideración podría sugerir una variación significativa de los componentes de la fuerza de trabajo masculina y femenina. Sin embargo, la tipificación por sexo de los empleos está sujeta a un cambio en virtud de que las oportunidades de trabajo son cambiantes, debido a que las economías se expanden y se diversifican. Una de las formas como se puede valorar hasta qué punto responde la fuerza de trabajo femenina ante las oportunidades cambiantes que resultan del desarrollo, es tratar de determinar cómo altera dicho desarrollo la participación desigual de la mujer en la estructura industrial.

Una meta empírica de esta investigación es establecer qué porciento de la variación de la ocupación femenina en los sectores de la industria puede deberse a la diferenciación regional. De manera específica este estudio examina cómo las variaciones regionales en el desarrollo socioeconómico influyen en la composición por sexos de la ocupación industrial en México, país que no sólo está sufriendo una profunda diferenciación interna, sino que se ha desarrollado considerablemente en los últimos tres decenios. ${ }^{4}$ Por lo tanto se consideran las relaciones entre la transformación estructural del empleo, la diferenciación socioeconómica y los cambios en la distribución de la fuerza de trabajo femenina. Se hace un análisis sectorial, basado en los datos del censo de 1970, con el fin de examinar la composición por sexos del empleo en los sectores industriales.

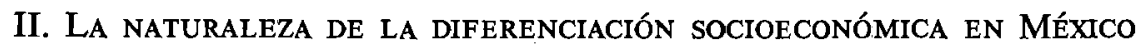

Una rápida industrialización y una diversificación estructural han sido los aspectos que distinguen a la economía mexicana desde 1930. Las con-

3 Nadia Haggag Youssef, Women and Work in Developing Societies, Berkeley. Institute of International Studies, Population Monograph Series, No. 15, 1974; Ester Boserup, Women's Role in Economic Development, Londres, George Allen and Unwin, 1970; Valerie Oppenheimer, "The Sex-Labeling of Jogs", Industrial Relations, Núm. 7, mayo de 1968, pp. 219-234; National Manpower Council, op. cit.

4 Clark W. Reynolds, The Mexican Economy. Twentieth Century Structure and Growth, New Haven, Yale University Press, 1970. 
secuencias e implicaciones del sostenido y relativamente reciente crecimiento económico de México pueden apreciarse mejor mediante un examen del curso de tal desarrollo, puesto que es este crecimiento el que afecta la transformación de la fuerza laboral y la composición por sexos de los empleos. En breve, los procesos de desarrollo de la industrialización y la modernización socioeconómica de México, que provocan la transformación de la fuerza laboral, han tenido como resultado una progresiva y marcada diferenciación regional entre cada una de las entidades federativas ${ }^{5}$ Por lo tanto, esta transformación proporciona un interesante caso de estudio para examinar las pautas del empleo por sector de la fuerza laboral femenina desde un punto de vista desarrollista. Es posible obtener un marco de referencia comparativo para analizar los patrones de la participación femenina por sectores, si se hace una clasificación de las 32 entidades mexicanas según índices escogidos de desarrollo socioeconómico. En este análisis transversal, cada estado representa grados variables de desarrollo socioeconómico.

Appendini et al. y Yates realizaron estudios para clasificar los estados de la República Mexicana con información obtenida en 1960, pero en vista de que este análisis se basa en las cifras de empleo de 1970, es preferible contar con un nuevo esquema de clasificación basado en datos más recientes. Los índices de desarrollo socioeconómico empleados para hacer la clasificación de las entidades mexicanas en 1970 fueron medidas de industrialización, urbanización, alfabetización de la mujer, número de obreros y producción per capita. ${ }^{6} \mathrm{Al}$ analizar los factores se confirmó solamente una dimensión subyacente que se designa como desarrollo socioeconómico. ${ }^{7}$ Por lo tanto, las entidades se clasificaron según su pun-

5 La asignación desigual de recursos durante las primeras etapas del desarrollo fue muy importante para determinar la pronunciada diferenciación socioeconómica por regiones, de manera tal que para 1960 había grandes discrepancias regionales dentro del país, como una de las características más sobresalientes. Véase K. A. Appendini, D. Murayama y R. M. Domínguez, "Desarrollo desigual en México, 1900 y 1960" Demografía y Economía, Vol. VI, Núm. 1, 1972, pp. 1-39; Paul Lamartine Yates, El desarrollo regional de México, México, Banco de México, Departamento de Investigaciones Industriales, 1961; Claudio Stern; Las regiones de México y sus niveles de desarrollo socioeconómico, México, Centro de Estudios Sociológicos, 1973.

6 Los indicadores empleados para construir una calificación de desarrollo para clasificar las entidades, se seleccionaron de modo que incluyeran tres aspectos generales de desarrollo: ecológico, social y económico. Cínco indicadores utilizados para representar estas dimensiones socioeconómicas de desarrollo son: 1) urbanización, definida como el porciento de la población total que reside en localidades de 5000 o más; 2) alfabetización entre las mujeres, definida como el porcentaje de mujeres de más de 10 años de edad que saben leer y escribir; 3) obreros, definido como la proporción de puestos que ocupan trabajadores no agrícolas; 4) producto per capita, defínido como la relación entre el producto total por entidad (en pesos) y la población total; 5) industrialización, definida como el porcentaje de la fuerza de trabajo total que se dedica a actividades manufactureras. Para mayores detalles sobre la selección de indicadores, definición y relación con los problemas del empleo de la mujer, véase Marta Tienda, "Diferencias socioeconómicas regionales y tasas de participación de la fuerza de trabajo femenina: el caso de México", Revista Mexicana de Sociología, Núm. 37, octubre-diciembre de 1975, pp. 911-929.

$7 \mathrm{La}$ validez de la calificación compuesta del desarrollo se refuerza en su asociación con los componentes individuales. Las correlaciones de orden son bastante ele- 
tuación en cada factor (las puntuaciones del índice de desarrollo aparecen en el anexo A) derivadas de la variación común de estos cinco indicadores.

Cuando se interpreta la relación existente entre el índice de desarrollo y la composición de la fuerza de trabajo femenina en la industria, es necesario reconocer que hay una diferencia entre el desarrollo socioeconómico experimentado por un país a través de los años y el rango de diferenciación que ocurre internamente en un determinado momento. Aún no está claro en qué consisten exactamente estas diferencias, pero ambas no pueden intercambiarse. Esto significa que las deducciones obtenidas con datos de sección transversal no pueden imponerse a los análisis longitudinales. Esta consideràción es pertinente en especial para el caso de México, ya que no existe una razón lógica para esperar que dos entidades se desarrollen de manera idéntica.

Aun cuando podría argüirse que ciertos requisitos son condiciones necesarias para que el proceso de desarrollo alcance impulso y movimiento continuo, es también válido el argumento contrario de que los hechos históricos que condicionan la dirección y el alcance de dicho proceso de desarrollo influyen de manera definitiva en la naturaleza y en el grado de la diferenciación regional durante una etapa determinada de desarrollo. En vista de estas consideraciones, no se pretende que los hallazgos que han de describirse puedan generalizarse a otros países 0 , inclusive, aun dentro de México en el futuro.

\section{CONCEPTUALIZACIÓN DE LA TRANSFORMACIÓN POR SECTORES DE LA FUERZA LABORAL}

Los cambios estructurales en la producción resultantes, y a la vez causantes, del impulso para seguir desarrollándose se manifiestan en la evolución de la producción industrial y en las estructuras ocupacionales. En particular, la baja relativa de empleo en el sector primario y el crecimiento en el sector terciario (Servicios) han sido un estímulo importante para aumentar la participación de la mujer en la fuerza de trabajo, tanto en los países en vías de desarrollo como en los desarrollados. Esta transformación sectorial es en gran parte responsable del aumento secular de la participación total de la mujer en el trabajo, porque es precisamente en el sector de servicios, en el que el empleo crece con gran rapidez, donde encuentran las mujeres mayores oportunidades de trabajo. ${ }^{8}$

vadas y van de 0.73 a 0.94 . Sin embargo, como este esquema de clasificación da muestras de cierta variación comparada con la clasificación de Yates y Appendini en 1960, es conveniente comentar las discrepancias. Por lo general existe una sorprendente semejanza entre los tres esquemas, en particular en los extremos mayor y menor del continuum. Los coeficientes de correlación de rango de Spearman, que miden la magnitud de la semejanza entre los rangos, son marcados y positivos $(0.86$ y 0.87) y ambos indican que existe congruencia entre los tres esquemas a pesar de que son un tanto diferentes en su construcción, y que la clasificación propuesta no es idiosincrática.

8 P. Bairoch y J. M. Limbur, "Changes in the Industrial Distribution of the World 
Las implicaciones sociales de la creciente actividad terciaria son de gran importancia ${ }^{9}$ y nunca dejaremos de tenerla en cuenta, pero a primera vista se perciben las enormes limitaciones de trabajar con una categoría demasiado heterogénea de industriales de servicios. Por ejemplo, en los países en vías de desarrollo los servicios personales son el aspecto principal del empleo "terciario", mientras que en las naciones desarrolladas predominan los servicios de educación, de salud y los legales y sociales. ${ }^{10}$ Llama la atención al estudiar la cambiante composición del empleo terciario contemplar la enorme variación de un sector a otro en los niveles de empleo en servicios tanto dentro de los países como al comparar un país con otro. A tal variación nos debemos dirigir explícitamente al delinear la relación entre la estructura del empleo en la industria y el desarrollo. Por lo tanto, para dilucidar cómo varía la participación de la mujer en los distintos sectores de la industria según el desarrollo socioeconómico, se necesita un esquema de la industria que sea más sensible a las influencias del desarrollo. Si los conceptos básicos de tal esquema son sólidos, será posible hacer análisis más diferenciados de las estructuras de la producción y del empleo y, lo que es más importante, al menos para nuestros propósitos, se contará con nuevas maneras de conocer la relación entre la naturaleza de la ocupación femenina y las distintas circunstancias socioeconómicas.

\section{Esquema de empleo por sector de la industria}

Browning y Singelmann ${ }^{\text {T1 }}$ han diseñado un esquema de la distribución por sexos en cada sector industrial. Los sectores que han elegido para trabajar son: 1) de extracción, que incluye a la agricultura, la silvicultura, la pesca y la minería; 2) de transformación, que incluye la fabricación, el procesamiento de minerales y de metales, y la construcción; 3) de distribución, que incluye la transportación, la comunicación y el comercio tanto al mayoreo como al menudeo; 4) de servicios al productor, que incluye la banca, los créditos, el financiamiento, los servicios legales y las compañías de seguros; 5) de servicio social, que incluye los servicios médicos, educacionales, de asistencia y gubernamentales; 6) de servicios personales, que incluye servicios de manejo de casa, de recreación, de restaurantes, de vivienda y de entrenamiento. En el análisis usaremos además una séptima categoría, 7) actividades insuficientemente especifica-

Labor Force, by Region, 1880-1960", International Labor Review, Núm. 98, septiembre de 1968, pp. 311-337.

9 Véase Joachim Singelmann, The Sectoral Transformation of the Labor Force in Seven Industrialized Countries, 1920-1960, Austin, Texas, tesis doctoral, 1974, capítulo VIII y Víctor R. Fuchs, The Service Economy, Nueva York, Columbia University Press, 1968, capítulo 8.

10 Harley L. Browning y Joachim Singelmann, The Emergence of a Service Society: Demographic and Sociological Aspects of the Sectoral Transformation in the USA, Report for the Manpower Administration, US Department of Labor, 1975.

11 Ibid., capítulo I. 
das, que no es parte del esquema de Browning-Singelmann. Esta categoría, que cubre la porción de la fuerza de trabajo donde el estatus ocupacional fue difícil de clasificar, es, por dos razones, particularmente importante para los países en vías de desarrollo. Primero, existen algunos casos en los que la población económicamente activa se ocupa genuinamente de actividades "ambiguas". Segundo, la oficina de censos ha reglamentado de manera insuficiente el proceso de clasificación.

En este esquema 'ocupacional, los sectores de extracción y de transformación corresponden de cerca con los esquemas convencionales tripartitas primario-secundario, pero el sector terciario se ha subdividido considerablemente. La razón por la que Browning y Singelmann subdividieron al sector de servicios en cuatro categorías, es la gran heterogeneidad de las industrias de servicio. A medida que aumenta la participación de la fuerza de trabajo en estas industrias, es menos útil el concepto de "servicios". Por esa razón, los autores del esquema dividieron el sector terciario convencional en cuatro sectores de servicios basándose en la función y en la clientela de los varios servicios.

Los servicios de distribución y al productor se relacionan esencialmente con la producción; la función de la mayoría de estos servicios es o bien distribuir los bienes o ayudar en su producción. Los otros dos sectores de servicio, personales y sociales, se abocan más a las personas, aunque con diferente énfasis. Los servicios personales están orientados principalmente al consumidor individual, mientras que los servicios sociales se ocupan más de la colectividad o la comunidad. Una de las características distintivas de los servicios sociales es el hecho de que las actividades que desarrollan no dependen en lo absoluto de la capacidad de los individuos para comprarlas, pues son el resultado de una decisión de la sociedad de mantener un cierto nivel de vida. Dado que todos los sectores de la industria, en especial el sector terciario, están sujetos a grandes cambios debidos al desarrollo económico, este esquema de la industria es más eficaz para detectar los cambios correspondientes en el empleo de mujeres dentro de un contexto de desarrollo.

\section{Composición del empleo por sexo en las industrias}

El empleo de mujeres en una entidad dada se puede expresar de la siguiente manera:

$$
N_{f}=\sum_{i=1}^{n} N_{i} f_{i}
$$

donde $N_{i}$ es el empleo total del sector industrial $i$ y $f_{i}$ es la proporción femenina en el sector industrial $i$.

Las diferencias en el empleo de mujeres en las entidades $\left(d N_{f}\right)$ pueden descomponerse para señalar cómo ocurren los cambios de empleo, de modo que: 


$$
d N_{f}=\sum_{i=1}^{n} N_{i} d f_{i}+\sum_{i=1}^{n} f_{i} d N_{i}
$$

Al expresar el cambio en el empleo de mujeres de esta manera, se demuestra cómo ocurren los cambios, es decir, ya sea alterando la participación femenina en cualquier sector dado, manteniendo constante el número de mujeres empleadas $\left(N_{i} d f_{i}\right)$, o cambiando el empleo total en un sector dado, mientras se mantiene constante la participación femenina $\left(f_{i} d N_{i}\right)$. A pesar de que muchos estudios se han referido a este último aspecto de los cambios en el empleo de mujeres, este estudio busca esclarecer cómo las variaciones internas del desarrollo socioeconómico en México influyen tanto en la participación media en el empleo por parte de las mujeres como en cada sector específico (véase el apéndice A).

El análisis empírico consta de dos etapas. Primero, la relación entre la participación de la mujer en el empleo total $\left(N_{f} / N\right)$ y la variable en el desarrollo socioeconómico se determina empleando cada entidad como unidad de análisis. Posteriormente se evalúa hasta qué grado afecta el desarrollo la participación femenina en el empleo entre los sectores particulares de la industria $\left(N_{i} f_{i}\right)$. No cabe duda que ambas medidas reflejan la composición del empleo por sexos, pero la segunda, que es específica de un sector de la industria, permite tal medida de manera más desglosada. Permite apreciar cómo varía con el desarrollo la desproporcionada representación femenina en la estructura industrial, en comparación con la representación masculina.

\section{REsultados}

La hipótesis general que subyace en la etapa inicial de este análisis se refiere a que existe una relación positiva entre el grado de desarrollo socioeconómico de una entidad y la proporción de la fuerza laboral femenina. Para comprobar esta hipótesis, se usa $\left(N_{f} / N\right)$ como una variable dependiente en dos modelos de regresión diferentes: un modelo multivariado en el que se utilizan los indicadores del desarrollo por separado en el lado derecho, y un modelo bivariado en el que se emplea un índice compuesto de desarrollo socioeconómico como predictor independiente. El modelo "índice" se emplea para entrelazar la dependencia lineal entre las variables independientes facilitando de esta manera la interpretación de los coeficientes de regresión.

Los resultados que aparecen en la parte superior del cuadro 1 apoyan la existencia de una relación positiva entre el empleo de mujeres y el grado de desarrollo socioeconómico. Como se esperaba, el modelo de indicadores múltiples da cuenta de un $15 \%$ adicional en la variación del empleo de mujeres en la ocupación dentro de México. Pero el $\mathbf{R}^{2}$ en el Modelo II es muy importante; es decir, aproximadamente un $63 \%$ de 
la variación en el empleo de mujeres puede atribuirse a diferencias en el grado de desarrollo que ha alcanzado cada entidad. En esencia por cada unidad de incremento en el índice de desarrollo hay un correspondiente $3 \%$ de aumento en la proporción de empleo que corresponde al sector femenino.

\section{Cuadro 1}

EFECTOS DEL DESARRollo SOBRE LA COMPOSICIÓN DEL EMPLEO POR SEXOS ${ }^{\mathrm{B}}$

\begin{tabular}{|c|c|c|}
\hline & \multicolumn{2}{|c|}{ Modelo } \\
\hline & 1 & II \\
\hline Producto per oapita & $\begin{array}{l}0.026 \\
(0.122)\end{array}$ & - \\
\hline Indus trialización & $\begin{array}{c}0.362 \\
(0.146)\end{array}$ & - \\
\hline Urbanizaeion & $\begin{array}{l}0.135 \\
(0.045)\end{array}$ & - \\
\hline Obreros & $\begin{array}{l}-0.311 \\
(0.171)\end{array}$ & - \\
\hline Alfabetismo entre mujeres & $\begin{array}{r}-0.054 \\
(0.053)\end{array}$ & - \\
\hline Calificación según fndice de desarrolloof & d/ & $\begin{array}{l}3.115 \\
(0.426)\end{array}$ \\
\hline$R^{2}$ & 0.786 & 0.633 \\
\hline
\end{tabular}

a Variable dependiente $=N_{\mathrm{f}} / N$, o proporción de mujeres empleadas en cada en tidad.

b Los números entre paréntesis corresponden a los errores estándar de los coeficientes.

c Apéndice A. Para su definición véase el texto y la nota 6.

d No incluida en la ecuación.

Una vez establecida la relación global, es pertinente desglosar este efecto examinando cómo varía la composición del empleo por sexos en cada sector de la industria de cada entidad. Sin embargo, debe establecerse primero la presencia de una asociación sistemática entre la participación media de la mujer en el empleo $\left(N_{f} / N\right)$ y la participación femenina en un sector específico $\left(N_{i} f_{i}\right)$ bajo circunstancias socioeconómicas variables. Este paso es esencial en vista de que en pruebas anteriores se confirmó la existencia de una asociación sistemática entre $\left(N_{f} / N\right)$ y el índice de desarrollo socioeconómico.

Se ha establecido la hipótesis de que en los niveles superiores de desarrollo se reduce la dispersión en el prociento de mujeres empleadas en los sectores de la industria en relación con su participación total en el empleo. De esto se puede inferir que el desarrollo reduce la importancia del sexo como mecanismo distributivo hasta el punto de que ocurre una convergencia entre $\left(N_{f} / N\right)$ y $\left(N_{i} f_{i}\right)$ debido a que los niveles de empleo femenino por sector específico se acercan más y más a la media. Para comprobar esta premisa se tomó una medida de dispersión, llamada $D$, en 
el nivel sectorial y medio de la participación femenina en el empleo, calculándose para todas las entidades e incorporadas en la regresión con las variables independientes empleadas en los modelos anteriores. El grado de dispersión en la ocupación femenina por sectores industriales se define como sigue:

$$
D=\sum_{i=1}^{z}\left[\left(N_{i} f_{i}\right)-\left(N_{f} / N\right)\right]^{2}
$$

donde $\left(N_{i} f_{i}\right)$ y $\left(N_{f} / N\right)$ son la participación femenina en el empleo del sector industrial i-ésimo y la participación femenina media entre todos los sectores, respectivamente, para cualquier estado dado. ${ }^{12}$

Los resultados que se presentan en el cuadro 2 revelan que el índice de desarrollo socioeconómico "explica" alrededor del 40 por ciento de la variación en la dispersión de la participación femenina en el empleo entre los sectores de la industria, en relación con la proporción media de mujeres que trabajan. Este coeficiente negativo sugiere que la cantidad de dispersión sectorial en el empleo femenino varía inversamente al desarrollo, lo cual refuerza aún más el argumento de que el desarrollo reduce la importancia del sexo en la distribución de mujeres en la estructura industrial.

\section{Cuadro 2}

EFECTOS DEL DESARROLLO EN LA DISPERSIÓN DE LA PARTICIPACIÓN DE LAS MUJERES EN EL EMPLEO POR SECTORES DE LA INDUSTRIA ${ }^{a}$

\begin{tabular}{|c|c|c|}
\hline & \multicolumn{2}{|c|}{ Modelo- } \\
\hline & I & II \\
\hline Producto per capita & $\begin{array}{c}-5.73 \\
(2.79)\end{array}$ & - \\
\hline Indus trial izaoion & $\begin{array}{l}75.27 \\
(33.09)\end{array}$ & - \\
\hline Urbanización & $\begin{array}{c}-25.52 \\
(10.36)\end{array}$ & - \\
\hline Obreros & -31.89 & - \\
\hline Alfabe tismo entre mujeres & -0.37 & - \\
\hline Calificación segün indice de desarrollo & c/ & $\begin{array}{c}-457.07 \\
(102.11)\end{array}$ \\
\hline $\mathbf{R}^{2}$ & 0.683 & 0.393 \\
\hline
\end{tabular}

a Como variable dependiente se utiliza la medida de dispersión descrita en la prueba.

b Los números entre paréntesis corresponden a los errores estándares de los coeficientes.

c No incluida en la ecuación.

12 No fue necesario dividir entre el número de sectores, pues se trataba de una constante en todas las entidades. 
Esta prueba no basta para determinar si la magnitud y el curso del efecto es uniforme en todos los sectores. Debido a la considerable variación intersectorial de la participación femenina, es necesario especificar aún más cómo la composición de sexos en cada sector de la industria se altera debido a las diferentes circunstancias socioeconómicas entre cada entidad en México. De hecho, se deben desglosar los resultados anteriores. Sin embargo, dado que $\left(N_{i} f_{i}\right)$ se relaciona con $\left(N_{f} / N\right)$, la primera no puede emplearse directamente para descubrir cuál es el efecto del desarrollo en el nivel de ocupación de la mujer por sectores, porque sería difícil establecer si el modelo solamente refleja las determinantes de $\left(N_{f} / N\right)$, o si de hecho explica las variaciones sectoriales específicas en el empleo femenino, es decir, $\left(N_{i} f_{i}\right)$.

$\mathrm{Al}$ analizar los sectores industriales individualmente en todas las entidades, es posible llevar un control de las diferencias estructurales resultantes de una variación económica regional entre las entidades. Para ajustar el nivel medio de empleo femenino con las variaciones interestatales, se diseñó una nueva variable que combina la proporción media de mujeres en la fuerza de trabajo con dicha participación en cualquier sector específico de la industria. Por lógica se deriva que como la proporción de mujeres en cualquier sector dado está en función de la participación total femenina en el empleo, el efecto de tal función debe eliminarse sistemáticamente de la variable que se va a probar. Este recurso "protege" a $\left(N_{f} / N\right)$ de inferencias sesgadas acerca de $\left(N_{i} f_{i}\right)$. Así, la variable dependiente empleada para examinar los efectos del desarrollo en la relación secorial del empleo femenino resulta: $\left[\left(N_{i} f_{i}\right)-\left(N_{f} / N\right)\right]$. De esta forma se "estandariza" cada uno de los sectores para estimar siete ecuaciones. Aunque la prueba de $\left(N_{i} f_{i}\right)$ es indirecta debido a los procedimientos de estandarización, los resultados son útiles para los fines propuestos porque la variable por verificar es la suma de las variables dependientes en la primera y tercera pruebas respectivamente, de tal modo que:

$$
\left(N_{f} / N\right)+\left[\left(N_{i} f_{i}\right)-\left(N_{t} / N\right)\right]=\left(N_{i} f_{i}\right)
$$

Dada la naturaleza derivada de la variable dependiente se garantiza una descripción de la interpretación sustantiva de los resultados. En esencia, esta prueba desglosa los resultados de la prueba anterior, que establecía que el desarrollo reduce la dispersión en el nivel de empleo de mujeres entre los sectores de la industria, en relación con su participación ocupacional total por entidad. Si se supone que $\left(N_{f} / N\right)$ es la participación esperada de empleo de mujeres, mientras que $\left(N_{i} f_{i}\right)$ es el porciento femenino real en cualquier sector dado, entonces la variable dependiente derivada $\left(N_{i} f_{i}\right)-\left(N_{f} / N\right)$ refleja la desviación entre la participación real de la mujer en el empleo y la esperada, o más generalmente, los desiguales niveles de empleo femenino en cada sector. Por lo tanto, un coeficiente negativo indicaría que el aumento en el nivel de desarrollo socioeconómico en una entidad reduce la desviación de la variable específica 


\section{Cuadro 3}

EFECTO DEL DESARROLLO EN LA COMPOSICIÓN DEL EMPLEO POR SEXOS Y POR SECTORES DE LA INDUSTRIA ${ }^{a}$

\begin{tabular}{|c|c|c|c|c|c|c|c|}
\hline Seotores b/. & $\begin{array}{l}\text { Produoto } \\
\text { por } \\
\text { napita }\end{array}$ & $\begin{array}{l}\text { Industria } \\
\text { lizacion }\end{array}$ & $\begin{array}{l}\text { Urbani- } \\
\text { zaoión }\end{array}$ & Obreros & $\begin{array}{l}\text { Alfabetio } \\
\text { mo entre } \\
\text { mujeres }\end{array}$ & $\begin{array}{l}\text { Callfica } \\
\text { oisnof }\end{array}$ & $a^{2}$ \\
\hline Bxtracoisn & $\begin{array}{c}-0.002 \\
(0.009) \\
-\end{array}$ & $\begin{array}{c}-0.149 \\
(0.109) \\
-\end{array}$ & $\begin{array}{c}-0.130 \\
(0.034) \\
-\end{array}$ & $\begin{array}{c}0.054 \\
(0.127) \\
-\end{array}$ & $\begin{array}{c}0.012 \\
(0.039) \\
-\end{array}$ & $\begin{array}{c}- \\
-2.609 \\
(0.274)\end{array}$ & $\begin{array}{l}0.801 \\
0.745\end{array}$ \\
\hline \multirow[t]{2}{*}{ Transformaot ón } & $\begin{array}{l}-0.038 \\
(0.028)\end{array}$ & $\begin{array}{l}0.211 \\
(0.333)\end{array}$ & $\begin{array}{c}0.073 \\
(0.104)\end{array}$ & $\begin{array}{l}-0.613 \\
(0.389)\end{array}$ & $\begin{array}{l}-0.121 \\
(0.120)\end{array}$ & - & 0.476 \\
\hline & - & - & - & - & - & $\begin{array}{l}-3.649 \\
(0.790)\end{array}$ & 0.406 \\
\hline \multirow[t]{2}{*}{ Distribuoion } & $\begin{array}{c}0.000 \\
(0.016)\end{array}$ & $\begin{array}{l}-0.157 \\
(0.192)\end{array}$ & $\begin{array}{l}-0.066 \\
(0.060)\end{array}$ & $\begin{array}{l}-0.312 \\
(0.224)\end{array}$ & $\begin{array}{l}-0.102 \\
(0.069)\end{array}$ & - & 0.780 \\
\hline & - & - & - & - & - & $\begin{array}{l}-4.442 \\
(0.439)\end{array}$ & 0.767 \\
\hline $\begin{array}{l}\text { Producoibn do } \\
\text { gorvịoios }\end{array}$ & $\begin{array}{l}-0.029 \\
(0.018)\end{array}$ & $\begin{array}{l}-0.161 \\
(0.216)\end{array}$ & $\begin{array}{l}-0.184 \\
(0.068)\end{array}$ & $\begin{array}{c}0.343 \\
(0.252)\end{array}$ & $\begin{array}{c}0.117 \\
(0.078)\end{array}$ & - & 0.557 \\
\hline . & $=$ & - & - & - & - & $\begin{array}{l}-2.320 \\
(0.590)\end{array}$ & 0.333 \\
\hline \multirow[t]{2}{*}{ Serviofos mootales } & $\begin{array}{l}-0.067 \\
(0.034)\end{array}$ & $\begin{array}{c}0.409 \\
(0.406)\end{array}$ & $\begin{array}{l}-0.090 \\
(0.127)\end{array}$ & $\begin{array}{c}0.078 \\
(0.475)\end{array}$ & $\begin{array}{c}0.172 \\
(0.147)\end{array}$ & - & 0.223 \\
\hline & - & - & - & - & - & $\begin{array}{l}-0.403 \\
(1.026)\end{array}$ & 0.005 \\
\hline \multirow[t]{2}{*}{ Sorvioios porsonalos } & $\begin{array}{l}-0.064 \\
(0.028)\end{array}$ & $\begin{array}{l}0.646 \\
(0.337)\end{array}$ & $\begin{array}{l}-0.237 \\
(0.106)\end{array}$ & $\begin{array}{l}-0.064 \\
(0.393)\end{array}$ & $\begin{array}{c}0.002 \\
(0.122)\end{array}$ & - & 0.633 \\
\hline & - & - & - & - & - & $\begin{array}{l}-3.706 \\
(1.044)\end{array}$ & 0.289 \\
\hline \multirow[t]{2}{*}{$\begin{array}{l}\text { Insuf lot on teren to } \\
\text { oapoolf leadoa }\end{array}$} & $\begin{array}{l}-0.020 \\
(0.021)\end{array}$ & $\begin{array}{c}0.144 \\
(0.248)\end{array}$ & $\begin{array}{l}-0.176 \\
(0.078)\end{array}$ & $\begin{array}{l}-0.481 \\
(0.290)\end{array}$ & $\begin{array}{l}-0.039 \\
(0.090)\end{array}$ & - & 0.794 \\
\hline & - & - & - & - & - & $\begin{array}{l}-5.962 \\
(0.585)\end{array}$ & 0.770 \\
\hline
\end{tabular}

a Los números entre paréntesis corresponden a los errores estándares de los coeficientes.

b La explicación respecto a la transformación de la variable dependiente aparece en el texto.

c Según el índice de desarrollo.

de un sector, en relación con el nivel medio de empleo de mujeres. Por lo tanto, a medida que aumenta el nivel de desarrollo socioeconómico se espera que disminya la diferencia entre la participación real de la mujer en el empleo y la esperada, en un sector industrial dado.

Los coeficientes de magnitud variable reflejan los cambios en la estructura ocupacional que acompañan a los procesos de desarrollo. Sin embargo, es ilusorio esperar que niveles elevados de desarrollo socioeconómico eliminarán por completo las diferencias sectoriales de empleo por sexo, aun cuando podrían disminuir bastante; la experiencia en los Estados Unidos testimonia este punto. ${ }^{13}$ Esto es necesariamente cierto

13 Valerie K. Oppenheimer, The Female Labor Force in the United States, Berkeley, Population Monograph Series, Núm. 5, en especial el capítulo 5. 
porque hay factores no económicos, tales como las prácticas de contratación discriminatorias, los trabajos designados por sexo, las actitudes negativas hacia la ocupación femenina, etc., que no están incluidos en las especificaciones y que influyen definitivamente en el grado $y$ en la naturaleza del mercado de oportunidades para la mujer.

Las estimaciones que aparecen en el cuadro 3 apoyan la hipótesis de que el desarrollo reduce las desviaciones entre el nivel real de la participación de la mujer en el empleo y el esperado, pero las magnitudes intersectoriales varían de manera considerable. En apariencia, la composición por sexos en el sector de servicio social no fue muy sensible a las variaciones en el desarrollo socioeconómico, según las operaciones realizadas para esta prueba. A continuación se incluye una interpretación sustantiva de estos resultados, en términos del marco conceptual sobre el que se basa la transformación estructural del empleo.

\section{Discusión}

La transformación sectorial de la fuerza de trabajo, de manera específica la salida del sector agrícola y el crecimiento paralelo de los servicios, se refleja en términos de la ocupación femenina frente a los cambios en los sectores de extracción y distribución. Para estos sectores de la industria, alrededor del $80 \%$ de la variación en el porciento de mujeres puede atribuirse a las diferencias socioeconómicas entre las entidades federativas de México. Este resultado está de acuerdo con nuestra premisa que relaciona los cambios estructurales del empleo con el desarrollo; es decir, conforme aumenta el grado de desarrollo socioeconómico, disminuye la participación de la mujer en la agricultura y aumenta su proporción en el sector de servicios.

En las economías tradicionales, la fuerza de trabajo femenina se ocupa de manera importante en las actividades agrícolas, aunque este hecho no resulte muy claro con una revisión superficial del Apéndice A. Sin embargo, se obtiene una imagen diferente al comparar las distribuciones totales. Por ejemplo, en los países de menor desarrollo, el sector de extracción absorbe entre el 18 y el $30 \%$ de todas las mujeres económicamente activas; en los países más desarrollados estas cifras son sólo de 1 a 12\%.14 Esto refleja en parte la tesis de Boserup, de que en las actividades de extracción, donde predomina la agricultura comercial, ${ }^{15}$ se prefiere la mano de obra masculina, y de que los hombres claramente dominan en las empresas minera y petrolera.

En general, parece ser que el desarrollo propicia un descenso continuo de las oportunidades de empleo primario para el sexo femenino. Sin

14 Marta Tienda, Economic Development and the Female Labor Force: The American Case, Austin, Texas, tesis de maestría, 1972, cuadro IV.3, p. 92.

15 E. Boserup, Women's Role in Economic Development, capítulo I. 
embargo, los cambios organizacionales que modifican las operaciones de este sector pueden estimular una demanda de mano de obra femenina en las formas modernas de empleo retribuido, como ocupaciones de oficinistas-técnicas que por regla general acompañan a la burocratización de las industrias primarias. Para confirmar esta aseveración, es necesario hacer un análisis paralelo de la estructura ocupacional sectorial; el Apéndice A nos proporciona algunas pruebas circunstanciales. Los datos muestran que en el Distrito Federal, área casi totalmente urbana, es mayor la proporción de mujeres empleadas en actividades extractivas en comparación con las zonas rurales. Es probable que muchas de estas mujeres ocupen puestos de "oficinistas" y no de extractores propiamente dichos.

En el sector de distribución, los aumentos en el índice de desarrollo socioeconómico propician una fuerte convergencia entre $\left(N_{f} / N\right)$ y $\left(N_{i} f_{i}\right)$ del orden de $4.4 \%$ por unidad. Debido a la sostenida tipificación por sexos de las industrias distributivas, se hace necesario examinar la división intrasectorial de la mano de obra por sexos, para poder interpretar de manera correcta el significado de este cambio. Los datos del censo muestran con claridad que las mujeres están sobrerrepresentadas en el sector comercial, en especial en el de comercio al menudeo, mientras que en la transportación continúa el dominio del sexo masculino. ${ }^{16}$ De aquí que los aumentos en la ocupación femenina en el sector de distribución se deban sobre todo a la expansión de las actividades comerciales, muy probablemente de las relacionadas con el comercio al menudeo. Al tener en cuenta estos hechos, queda claro no sólo que el desarrollo afecta de manera desigual al empleo de mujeres sino que las influencias intrasectoriales tampoco son uniformes. Por lo tanto, es difícil juzgar si los logros en este sector realmente significan un avance en el estatus económico de la mujer.

El empleo de mujeres en las actividades transformativas se extiende relativa y absolutamente al tiempo que aumenta el desarrollo, pero, por lo general, no tan rápido como el empleo en el sector de servicios. No obstante, estos resultados muestran que los logros en este sector son mayores que aquellos en cualquiera de los sectores de servicios. De manera específica, los mayores índices de desarrollo socioeconómico explican alrededor del $41 \%$ de la variancia en dispersión entre el nivel real y el esperado del empleo de mujeres en el sector transformativo, mientras que la cifra respectiva para los servicios al productor y los personales es de alrededor del $30 \%$, y considerablemente inferior en servicios sociales.

Estos resultados pueden parecer contradictorios frente a la afirmación de que la proporción de mujeres empleadas en las actividades transformativas permanece estable o disminuye, a niveles de desarrollo más elevados. Pero esta afirmación justifica la calificación. El descenso en el empleo femenino en las industrias manufactureras es especialmente pro-

16 México, Dirección General de Estadística, IX Censo de Población, Resumen General Abreviado, México, 1972, cuadro 27. 
bable en donde predominan una industrialización basada en el capital. Primero, debe señalarse que la composición de empleo por sexo en las industrias de servicios favorece constantemente a la mujer en todas las entidades, al margen del nivel de desarrollo (véase el Apéndice A). Esto no es válido para el sector de transformación. Los resultados reflejan el hecho de que las oportunidades de empleo para las mujeres aumentan a una tasa más rápida en el sector de transformación que en cualquier sector de servicios, a excepción del sector de distribución. Sin embargo, el efecto de la diferenciación socioeconómica en el empleo total del sector de servicios es con gran probabilidad mayor que en el empleo del sector de transformación.

Segundo, debe reconocerse que los cálculos para el sector manufacturero pueden estar sesgados hacia arriba porque, para las mujeres, las actividades de transformación abarcan una amplia gama de tecnología que van de la industria artística y artesanal familiar al trabajo administrativo en las grandes empresas. La producción en masa puede disminuir el empleo femenino cuando reduce el número de mujeres que trabajan por su cuenta en la industria artística y artesanal, debido a que sus productos por lo general no pueden competir con artículos producidos en masa. En la medida en que los procedimientos de producción modernos introducen tecnologías intensivas en el uso de capital, disminuye la demanda de trabajadores no calificados. $Y$ para las mujeres esto casi siempre significa un rango más limitado de oportunidades de empleo sectorial ya que por lo general las mujeres cuentan con menor experiencia, capacitación y otras formas de inversión en recursos humanos, si se les compara con los hombres. Por consiguiente, al realizarse una producción moderna las mujeres son a menudo sustituidas por hombres o por máquinas.

Puede argüirse que una parte de la variancia no explicada puede atribuirse a las discrepancias fundamentales en el tipo y la escala de la producción de transformación en las entidades mexicanas. A este respecto, es importante anotar que, con una sola excepción, la participación femenina en la ocupación del sector de transfomación para las nueve entidades menos desarrolladas, es mayor que la media global para la entidad $\left(N_{f} / N\right)$. Por otro lado, sólo dos incidencias de este patrón aparecen en cada una de las categorías de desarrollo alto y mediano, lo cual es testimonio de la importancia de las diferencias en el grado de capitalización entre las entidades de México.

Un examen más minucioso de la composición ocupacional intraindustrial también revela notable variación entre las entidades. Por ejemplo, en Coahuila, un estado altamente desarrollado, de todas las mujeres empleadas en fábricas, el $25 \%$ es personal administrativo y el $56 \%$ son jornaleras, en su mayor parte obreras. En contraste, en Oaxaca, un estado económicamente pobre, sólo el $1 \%$ de las mujeres que trabajan en las fábricas son parte del personal administrativo, mientras que el $90 \%$ están 
clasificadas como obreras. ${ }^{17}$ Sin duda, las diferencias en la naturaleza de la industria de transformación determinadas por el tipo de la empresa y de la sofisticación tecnológica, la estructura ocupacional y el nivel de capacidad requerido son definitivamente condicionadores de cómo va a reaccionar el sector de transformación ante el empleo de mujeres. La investigación mutua deberá considerar también estos factores cuando examine de qué manera influye la modernización de la producción en las tendencias del empleo de mujeres.

Entre los sectores de servicios al productor y personales, el índice de desarrollo explica sólo entre el 29 y el 33\% de la variación entre el nivel real y el esperado de ocupación femenina. Para entender estos resultados, es importante distinguir entre la cantidad y la calidad de la mano de obra. Donald Keesing, ${ }^{18}$ un estudioso de la historia económica de México, apoya este argumento haciendo notar que las actividades de servicio mexicanas recientemente han sufrido una transformación profunda cuando se incrementó de manera considerable la producción por trabajador. Aunque los servicios por lo general se basan sobre todo en el trabajo, los niveles de capacidad requerida dependen de manera significativa del nivel de desarrollo económico de la región. Señala, por ejem plo, que en países más avanzados, los servicios se centran mucho en la capacidad, de tal modo que cerca de un tercio del empleo en el sector está constituido por personal técnico y profesional..$^{1 \theta}$

Otra consideración digna de señalarse acerca del desarrollo y del proceso de terciarización es la sobresaliente pauta que resulta de la distribución intra e intersectorial por sexos. No sorprende que en 1970, el $45 \%$ de todas las mujeres mexicanas económicamente activas se dedicaran a alguna ocupación en el renglón de servicios, en comparación con el $13 \%$ del total de los hombres empleados. Sin embargo, esto oculta el hecho de que dentro de los sectores de servicios algunas actividades están casi en exclusiva dominadas por las mujeres. De manera específica, una gran parte del incremento en la ocupación femenina en los servicios personales se atribuyen a una creciente demanda por servicios domésticos. El impulso para esta creciente demanda se debe en gran parte al aumento en el nivel de ingresos de la naciente clase media. Dado que los servicios personales se caracterizan por estar orientados hacia el consumidor individual, éstos responden más a las fuerzas de oferta y demanda que a los servicios sociales: ${ }^{20}$

Los servicios sociales se encuentran relacionados con los individuos a través de la demanda colectiva y dado que con frecuencia estos servicios

17 México, Dirección General de Estadística, IX Censo de Población, Resumen General, México, 1972, cuadro 39.

18 "Structural Changes Early in Development; Mexico's Changing Industrial and Occupational Structure from 1895-1950", Journal of Economic History, Núm. 37, diciembre de 1969, pp. 716-738.

19 lbid., pp. 734-738.

20 Singelmann, The Sectoral Transformation of the Labor Forçe, p. 46. 


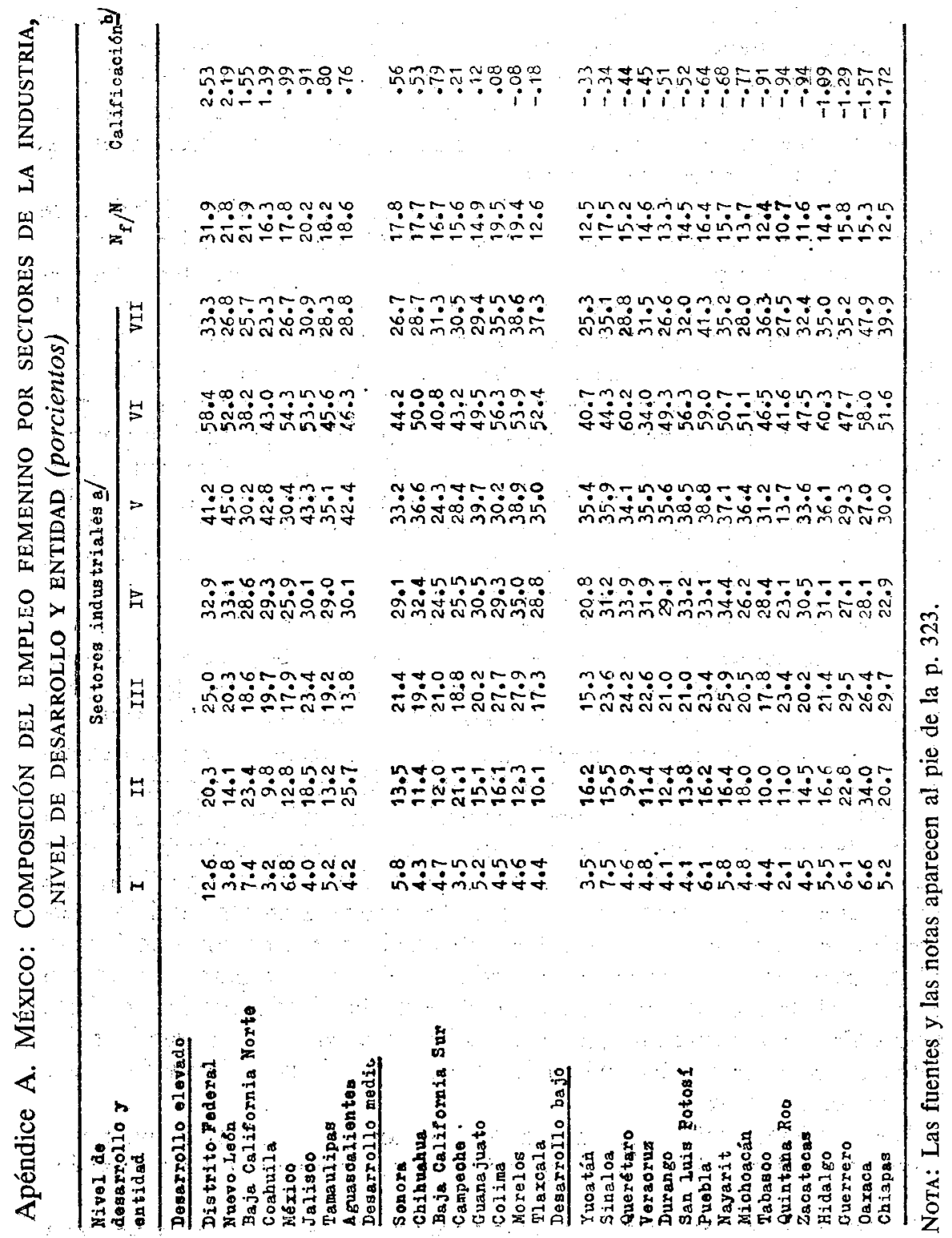


están subsidiados por el gobierno, su crecimiento está vinculado positivamente con la riqueza de un país. Por lo tanto, debido a la considerable reglamentación institucional, este sector industrial de empleo es el menos afectado por los ciclos comerciales y las cambiantes condiciones económicas. ${ }^{21}$ Esto explica en parte por qué las estimaciones empíricas para el sector de servicio social fueron las peores en términos de variación explicada entre los niveles reales y los esperados de empleo de mujeres y de la tasa de cambio con aumentos en el nivel de desarrollo. Los aspectos que se refieren a la tipificación de empleos por sexo y a los requisitos de experiencia sirven también para explicar este resultado.

La mayoría de los servicios sociales, especialmente en los puestos paramédicos, educacionales y de bienestar social, por tradición han sido empleos para el sexo "femenino" y siguen siéndolo; el desarrollo económico no afecta este renglón ni fácil ni gradualmente. Más aún, los puestos "profesionales" en los servicios sociales abarcan una amplia gama de requisitos de capacitación y habilidad. Por ejemplo, O'Hagan menciona que "en México... después de seis años de instrucción primaria y tres de secundaria, un estudiante puede cursar la carrera de normalista, que consta de tres años, y estar calificado para enseñar en la escuela primaria". ${ }^{22}$ La práctica estándar de clasificación y de presentación de los censos agrupa estos "semiprofesionales" junto con otros profesionales de servicios más capacitados, como por ejemplo, los doctores, los académicos y el personal administrativo. Esto también debilita la utilidad analítica de un sector de la industria tan heterogéneo desde el punto de vista ocupacional.

Antes de finalizar esta discusión se justifica una descripción sobre el efecto del desarrollo en el sector de actividades insuficientemente especificadas. Las estimaciones empíricas fueron las mejores para este grupo de actividades, a pesar de lo poco claro de su estatus. Quizá la importancia de una categoría de empleo tan vaga y heterogénea disminuirá con el tiempo, pero la probabilidad de que esto suceda no depende tanto del desarrollo per se como de que cambien las prácticas de clasificación y presentación de los resultados de los censos. Los resultados indican que las entidades menos desarrolladas experimentan aun mayores dificultades

21 Ibid., p. 46.

22 Maryann Joan O'Hagan, Distribution of the Economically Active Women in Latin America According to Type of Industry and Occupation, Austin, Texas, tesis de maestría, 1968, p. 43.

Fuentes del cuadro del apéndice A: Dirección General de Estadística, IX Censo General de Población. Resumen General Abreviado, cuadro 27. Para la califícación del nivel de desarrollo véase Marta Tienda, "Diferencias socioeconómicas regionales y tasas de participación de la fuerza de trabajo femenina: El Caso de México", cuadro 1, Revista Mexicana de Sociología, Núm. 37, octubre-diciembre de 1975 , pp. $911-929$.

a I: extracción; II: transformación; III: distribución; IV: producción de servicios; V: servicios sociales; VI: servicios personales; VII: insuficientemente especificados.

b Según índice de desarrollo. 
para proporcionar y clasificar los datos sobre la fuerza de trabajo que las entidades más desarrolladas.

\section{RESUMEN Y CONCLUSIONES}

Este estudio intenta principalmente descubrir hasta qué punto el desarrollo amplía la esfera de acción de los puestos disponibles para las mujeres por medio de su participación sectorial. De manera específica se ha demostrado que la participación femenina en el empleo está sin duda relacionada con altos niveles de desarrollo en las entidades de México, a pesar de que los hallazgos también han confirmado que el desarrollo no nulifica la importancia del sexo como un mecanismo distributivo. En general, los resultados empíricos muestran que los altos niveles de desarrollo socioeconómico entre las entidades mexicanas propician una convergencia entre la participación femenina en el empleo, tanto en términos medios como por sectores específicos. A continuación, se analizó el impacto del desarrollo en la participación del empleo por sexos y se esespecificó la magnitud de la convergencia para cada uno de los sectores de la industria.

Aunque los hallazgos sugieren que el grado al que la fuerza de trabajo se estratifica por sexo en la dimensión sectorial se relaciona de manera importante con la diferenciación socioeconómica interna de México, con base en las pruebas presentadas, es prematuro concluir que se ha mejorado la posición relativa de las mujeres.

De hecho, una estructura industrial altamente estratificada por sexo describe el patrón interno de colocación de México. La estructura ocupacional aún no se ha examinado en detalle, pero los pocos ejemplos citados indican que la estructura ocupacional también se encuentra dividida por sexos.

Nuestro análisis sectorial basado en la experiencia de un solo país ilustra cómo cambia el empleo femenino con los procesos de diferenciación. En este análisis se utilizaron las entidades federativas mexicanas individualmente como sustitutos para los "grados" de desarrollo, pero el estudio actual no es un sustituto para análisis seriados. Esto es, no puede suponerse que el desarrollo tendrá el mismo efecto en la distribución de la fuerza de trabajo femenina a través de los sectores entre todas las entidades al cabo del tiempo, dado que la relación entre la naturaleza y la extensión de la actividad de la fuerza de trabajo femenina en México depende, en esencia, de las peculiaridades del proceso de desarrollo de cada entidad.

Aún queda mucho por hacer para mostrar cómo la diferenciación regional interna que resulta de una distribución desigual de los recursos económicos va a afectar la actividad de la fuerza de trabajo femenina. Al parecerer seguirán los aumentos en el nivel de ocupación femenina en 
todas las entidades mexicanas, pero esto en gran parte depende del ritmo y del alcance de la urbanización y la expansión concomitante de los sectores de servicios, pues éstos son en particular importantes para ampliar las oportunidades de trabajo para el sexo femenino en el futuro. La dispersión en las tasas de participación femenina entre las entidades de mayor y menor desarrollo puede disminuir con el tiempo, aun cuando la magnitud de la diferenciación económica entre las entidades continúe aumetando, puesto que el intenso proceso de terciarización no es exclusivo de las áreas desarrolladas. Más bien, el crecimiento de los servicios en las áreas en desarrollo ha sido notablemente diferente del que experimentaron los países industriales en el siglo $\mathrm{xrx}^{23}$

La continua importancia de la clasificación por sexo de los trabajos también contribuirá a un probable aumento futuro de la actividad de la fuerza de trabajo femenina en México. A pesar de que a menudo se mencionan las consecuencias negativas de esta práctica, es decir, la disminución del rango de oportunidades de empleo para la mujer, deben también señalarse las características positivas. De manera específica, una ventaja importante de la clasificación de los trabajos por sexo es que "se reservan" para las mujeres puestos que podrían ser ocupados por los hombres en condiciones de un mercado de competencia que fuera perfecto.

Los análisis longitudinales ayudarán a esclarecer estas proyecciones, pues aún no está claro exactamente cómo va a afectar el desarrollo la participación y ocupación de la fuerza de trabajo femenina en el contexto de la extensa diferenciación socioeconómica regional. Las investigaciones del futuro deberán examinar a la industria por distribuciones ocupacionales para especificar esta asociación con más precisión que la obtenida con los análisis de corte transversal. Más aún, a pesar de la pobre teoría económica que relaciona la actividad de la fuerza de trabajo femenina con el desarrollo y la 'diferenciación socioeconómica, es preciso desglosar de qué manera se altera el grado de segmentación del mercado por sexo como resultado del progreso socioeconómico. En breve, tendrán prioridad para futuras investigaciones los estudios que se refieran al problema de cómo el desarrollo va incorporando a las mujeres a la fuerza de trabajo y los análisis que identifiquen por medio de qué mecanismo de distribución se llegará a tal incorporación.

${ }_{23}$ Harley L. Browning, "Some Problematics of the Tertiartization Process in Latin America". Trabajo presentado al XL Congreso de Americanistas, Roma, Italia, 1972. 